IAU Colloquium 164: Radio Emission from Galactic and Extragalactic Compact Sources

ASP Conference Series, Vol. 144, 1998

J. A. Zensus, G. B. Taylor, \& J. M. Wrobel (eds.)

\title{
The Injector and the AGN Core
}

\author{
L. I. Matveenko
}

Space Research Institute, Moscow 117810, Russia

\section{I. K. Pauliny-Toth}

Max-Planck-Institut für Radioastronomie, D-53121 Bonn, Germany

Abstract. The fine core-jet structure of $3 \mathrm{C} 345$ and its low-frequency variability are discussed.

One of the problems in studies of the fine structure of quasars is the identification of the compact components at different frequencies and epochs. A reference feature is the core, which is clearly discernible at high frequencies, but is very weak at low frequencies.

Quasar nuclei are surrounded by an ionized medium emitting narrow lines, of size $10^{21} \cdot \mathrm{L}_{46} \mathrm{~cm}\left(\mathrm{~L}_{46}\right.$ is the UV luminosity in $\left.10^{46} \mathrm{erg} \cdot \mathrm{s}^{-1}\right)$ and with electron density $10^{4-6} \mathrm{~cm}^{-3}$ (Netzer 1987). For the quasar $3 \mathrm{C} 345$ the size of the ionized region is $10^{20} \mathrm{~cm}(33 \mathrm{pc})$ or 8 mas. The UV radiation ionizes the gas and changes the emission-line intensities. The timescale is several days to several years.

The injector of relativistic particles is the core itself. A flow of the relativistic particles pushes the surrounding medium apart and produces a channel bounded by the cocoon wall of the ionized medium (Begelman et al. 1984). The relativistic plasma flow moves with a velocity $\sim c$ and generates a magnetic field that focuses the plasma into fine filaments which wind around the axis, forming a helical jet. Individual plasma knots also move along the helix (Krichbaum et al. 1993). The period of the $3 C 345$ helix is $T=1.4 \cdot R^{1.1}$ mas ( $R$ is the distance from the injector) and its diameter is $\theta=0.45 \cdot \log R$ mas, (Matveenko et al. 1992). The period $\mathrm{T} \sim \mathrm{R}$ and the timescale of emission of the relativistic electrons is $\mathrm{t} \geq 10^{6} \mathrm{yr}$.

In this case, the observed peculiarities of the core and compact components reflect the working regime of the injector, as well as the observed radio variability at both high and low frequencies. The electron density in the cocoon wall falls with increasing distance from the injector, so that the wall becomes more transparent. Therefore, the injector itself or the nearest part of the jet can only be seen at the high radio frequencies.

The recombination time $\mathrm{t}_{r}=10^{5} \cdot \mathrm{N}_{\mathrm{e}}^{-1} \mathrm{yr}$ of the absorbing medium cannot exceed the time of the low frequency variability, i.e., about a year and $\mathrm{N}_{\mathrm{e}} \sim$ $10^{5} \mathrm{~cm}^{-3}$. For the optical depth $\tau \sim 1$ the wall thickness is $1=7 \cdot 10^{-4} \mathrm{pc}$. A region of the low-frequency variability (at $\lambda=49 \mathrm{~cm}$ ) is located at about 16 mas from the core, where the cocoon becomes transparent and corresponds to the nearest part of the jet (Rantakyrö et al. 1995, Matveenko et al. 1996). The low frequency variability arises from the changes of the visible size of the compact region. Its brightness temperature is practically unchanged and is $T_{b} \sim 10^{12} \mathrm{~K}$.

The 3C 345 compact region ("core") has a brightness temperature $T_{b} \sim$ $0.01 \cdot T_{\text {bmax }}$ or $10^{10} \mathrm{~K}$. The drop by a factor of 100 can be due to absorption, which corresponds to an optical depth of 5 . The absorption in the cocoon at $\lambda 6$ $\mathrm{cm}$ would be $\leq 20 \%$ and the wall is essentially transparent at millimeter and short centimeter wavelengths. 
The 3C 345 compact components are spread along the jet in a region within 8 mas of the core (Zensus 1995, Brown et al. 1994). At high frequencies, the cocoon is transparent and does not distort the observed structure, however, it can affect parameters of the polarized emission. In the mid-1980s the polarized emission of $3 \mathrm{C} 345$ at $6 \mathrm{~cm}$ increased simultaneously with the total emission, but, reaching a certain level, began to decrease, while the total emission continued to rise and then everything occurred in reverse order. The region of peak polarized emission was 4.5 mas from the bright compact component (Brown et al. 1994), corresponding to 7 mas from the core. The position angle varies with time and from component to component. The rotation measure of this region is $\mathrm{RM}=29 \mathrm{rad} \cdot \mathrm{m}^{-2}$ and the degree of polarization is $4 \%$. The electron density of the cocoon wall is $\mathrm{N}_{\mathrm{e}}=10^{5} \mathrm{~cm}^{-3}, \mathrm{l}=7 \cdot 10^{-4} \mathrm{pc}$, and $\mathrm{B}_{\|} \sim 0.5 \mathrm{mG}$. The magnetic field and the electron density vary with distance: $\mathrm{N}_{\mathrm{e}} \sim\left(\mathrm{r} / \mathrm{r}_{o}\right)^{-2}$, $\mathrm{l} \sim\left(\mathrm{r} / \mathrm{r}_{\mathrm{o}}\right)$ and $\mathrm{RM} \sim\left(\mathrm{r} / \mathrm{r}_{\mathrm{o}}\right)^{-3}$. In this case the core region $\left(\mathrm{r}_{\mathrm{o}} \sim 0.5\right.$ mas $)$ has $\mathrm{RM}=8 \cdot 10^{4} \mathrm{rad} \cdot \mathrm{m}^{-2}, \mathrm{~B}=0.1 \mathrm{mG}, \mathrm{N}_{\mathrm{e}}=10^{6} \mathrm{~cm}^{-3}$. These parameters explain the increase of polarization with distance from the core. The degree of polarization depends on the bandwidth $P / P_{o}=\sin (2 X \Delta f / f) /(2 X \Delta f / f), X=R M \cdot \lambda^{2}$. The observations of $3 \mathrm{C} 345 \mathrm{had} \Delta \mathrm{f}=28 \mathrm{MHz}$, (Brown et al. 1994). In this case, the observed polarization increases from $4 \%$ near the core to $15 \%$ corresponding to $\mathrm{RM}=6 \cdot 10^{4} \mathrm{rad} \mathrm{m}^{-2}$, which closely matches the estimate given above. The rise in the total radio emission from the core at $6 \mathrm{~cm}$ observed in the mid-1980s, accompanied by a "dip" in the polarization is explained by an increase of the rotation measure. During 1984-1986 the polarized emission fell from 3-4\% to $1 \%(3-4$ times $)$. For $\mathrm{X}=2.5 \mathrm{rad}$, an increase in this value of only $0.5 \mathrm{rad}$, or in RM by a factor of 1.2 is sufficient to account for the observed effect. At the same time, the transparency of the screen will change from $\tau=0.058$ to 0.07 or the absorption increase by a factor of 1.07 . The spectra of the radio outbursts and of the emission of the core are steep, with $\alpha \geq 3$, at low frequencies. "Negative" outbursts at low frequencies are observed. The steep cutoff in the spectrum is the result of the additional absorption in the cocoon wall.

Acknowledgments. LM is grateful to the IAU Colloquium organizers and L. Kogan for hospitality and financial support.

\section{References}

Begelman, M. C., et al. 1984. Rev. Mod. Phys., 56, 255-351.

Brown, L. F., et al. 1994. ApJ, 437, 108-121.

Krichbaum, T. P., et al. 1993. $A \& A, 275,375-389$.

Matveenko, L. I., et al. 1992. Soviet Ast. Lett., 18, 379-391.

Matveenko, L. I., et al. 1996. $A \& A, 312,738-744$.

Netzer, H., 1987. in NATO Adv. Sci. Ser., Math. Phys. Sci. 208: Astrophysical Jets And Their Engines, ed. W. Kundt (Dordrecht: Reidel), 1987. 103-124.

Rantakyrö, F. T. et al. 1995. $A \in A, 293,44-55$.

Zensus, J. A., et al. 1995. $A p J, 443,35-53$. 